\title{
Navigated transcranial magnetic stimulation for mapping the motor cortex in patients with rolandic brain tumors
}

\author{
Satoshi Takahashi, M.D., Ph.D., Peter Vajkoczy, M.D., Ph.D., and Thomas Picht, M.D. \\ Department of Neurosurgery, Charité-Universitätsmedizin Berlin, Germany
}

\begin{abstract}
Object. Navigated transcranial magnetic stimulation (nTMS) is a novel technology in the field of neurosurgery for noninvasive delineation of cortical functional topography. This study addresses the spatial accuracy and clinical usefulness of nTMS in brain tumor surgery in or near the motor cortex based on a systematic review of observational studies.

Methods. A systematic search retrieved 11 reports published up to October 2012 in which adult patients were examined with nTMS prior to surgery. Quality criteria consisted of documentation of the influence of nTMS brain mapping on clinical decision making in a standardized prospective manner and/or performance of intraoperative direct electrical stimulation (DES) and comparison with nTMS results. Cross-observational assessment of nTMS accuracy was established by calculating a weighted mean distance between nTMS and DES.

Results. All studies reviewed in this article concluded that nTMS correlated well with the "gold standard" of DES. The mean distance between motor cortex identified on nTMS and DES by using the mean distance in 81 patients described in 6 quantitatively evaluated studies was $6.18 \mathrm{~mm}$. The nTMS results changed the surgical strategy based on anatomical imaging alone in $25.3 \%$ of all patients, based on the data obtained in 87 patients in 2 studies.

Conclusions. The nTMS technique spatially correlates well with the gold standard of DES. Its functional information benefits surgical decision making and changes the treatment strategy in one-fourth of cases. (http://thejns.org/doi/abs/10.3171/2013.1.FOCUS133)
\end{abstract}

\section{KEY WoRdS • motor cortex mapping • navigated brain stimulation • navigated transcranial magnetic stimulation • brain tumor}

$\mathrm{T}$ He goal of brain tumor surgery is to maximize the extent of tumor resection while preserving function. To achieve this goal is challenging, especially in glioma surgery, since these operations often involve structures that potentially carry essential function. ${ }^{18}$ To minimize the risk for new neurological sequelae as well as the risk of leaving residual tumor, precise knowledge of the individual functional topography is indispensable. Because of natural anatomical variation between all people, displacement of familiar anatomical landmarks by the tumor, and tumor-induced plastic reorganization of functional areas, functionally relevant brain tissue cannot be reliably predicted from standard anatomical imaging alone. Thus, it is essential to obtain case-specific knowledge of the location of functionally essential brain tis-

\footnotetext{
Abbreviations used in this paper: $\mathrm{APB}=$ abductor pollicis brevis; $\mathrm{DCS}=$ direct cortical stimulation; DES $=$ direct electrical stimulation; $\mathrm{fMRI}=$ functional MRI; MEG = magnetoencephalography; MEP = motor evoked potential; nTMS = navigated transcranial magnetic stimulation; $\mathrm{TA}=$ tibialis anterior.
}

sue. Intraoperative brain mapping by means of DES has been so far the most accurate and reliable gold standard to obtain such knowledge. However, if we could obtain functional maps that delineate resectable versus nonresectable brain tissue outside the operating theater with the same accuracy and reliability as DES, it would be of great use.

Transcranial magnetic stimulation is the only modality that is analogous to DES in that it allows for electrical stimulation of the brain and observation of the induced effect (Fig. 1). Although TMS was introduced in clinical neurology in 1985, ${ }^{1}$ its use in neurosurgery has been sporadic, and only recently an increasing number of reports from neurosurgical institutions on the use of nTMS for brain mapping in patients with rolandic brain tumors have been published. $3,4,6,8-11,13,14,21$

In the present article, based on a review of previous reports evaluating the spatial accuracy of nTMS in comparison with the gold standard of DES and/or assessing the clinical impact of the method, we address the clinical utility of nTMS in rolandic brain tumor surgery. 

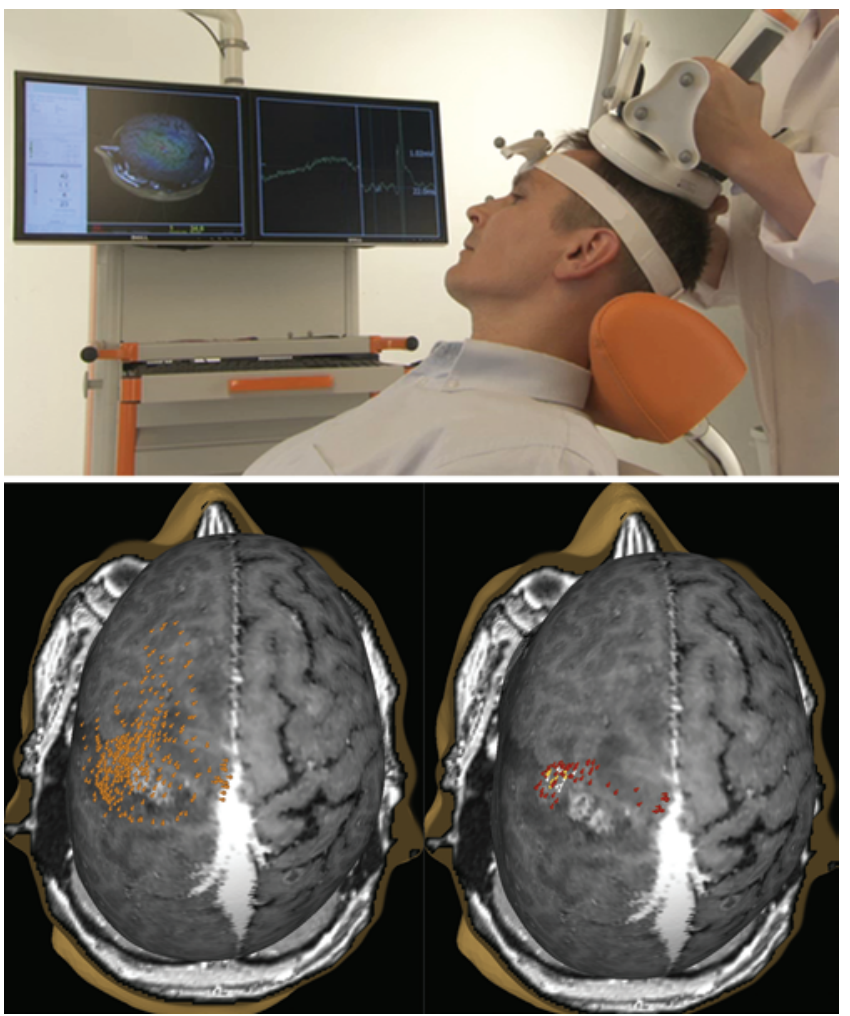

FIG. 1. Upper: The "E-field navigation" TMS system in use. Reflective spheres are attached to the patient's head (modified elastic headband) and to the TMS coil. The stimulator coil is shown placed against the patient's head. The motor output is recorded by surface electrodes attached to the face, arm, and leg. The anatomical map of the patient's brain and the MEP output tracings are shown on the computer screens in the upper left corner. Lower: A 3D reconstruction of an MRI study, navigational view, obtained in a 63-year-old woman suffering from a mild hemiparesis of her right side due to a tumor in the central region of the left hemisphere. All spots stimulated with nTMS on the left hemisphere are displayed in the left panel. The image in the right panel displays only the spots where a muscle response was observed (MEP > $50 \mu \mathrm{V}$ peak-to-peak amplitude). Three different hand muscles (APB, first dorsal interosseus, and abductor digiti minimi) and 1 leg muscle (TA) were recorded. The color coding corresponds to the intensity of the response, in which red indicates small responses (MEP 50-499 $\mu \mathrm{V}$ ), yellow indicates medium responses (MEP 500-999 $\mu \mathrm{V}$ ), and white indicates large responses (MEP $\geq 1000 \mu \mathrm{V}$ ). This mapping makes it evident that the precentral gyrus has been displaced frontally and that the center of gravity for the hand muscle representation is located immediately adjacent frontolaterally to the tumor. The responses close to the midline are from the leg (TA).

\section{Methods}

To establish the clinical usability of nTMS, it must be assessed relative to DES, because DES has been the gold standard method of brain mapping for many years.

For that purpose we have conducted a review of the literature up to October 2012. In our review we have evaluated the studies that have assessed the ability of nTMS to identify the motor cortex and delineate the cortical representation of individual muscles. The search terms we used on PubMed were "transcranial magnetic stimulation," "TMS," "direct cortical stimulation," "direct electrical stimulation," "DCS," "DES," "motor cortex," "M1," and "brain tumo(u)r." We reviewed the abstracts of those studies, and if they reported on evaluation of patients with rolandic tumors by using both nTMS and DES and/or if they assessed the impact of nTMS on surgical decision making, then we extracted information from the report.

\section{Literature Review}

A total of 11 studies meeting criteria written in the Methods section were identified (Table 1). For mapping of the motor cortex, most studies used a biphasic TMS pulse (250-280 $\mu$ sec pulse length) from a figure-eight coil with an outer diameter of $70 \mathrm{~mm}$ applied at $110 \%$ of the resting motor threshold and a maximum frequency of 0.25 Hz. ${ }^{2-5,7-9,12,14-17,20,21}$ For lower-extremity stimulation the intensity was adapted on an individual basis.

The first study to compare spatial accuracy of nTMS to that of DES for evaluating the motor cortex was done by Krings et al. ${ }^{10}$ They used a mechanical stereotactic arm for navigating the TMS system. Areas of motor responses identified by both nTMS and DES were compared in 2 patients with rolandic tumors. The discrepancy between nTMS and DES maps reported in the paper was never more than $1 \mathrm{~cm}$. The major limitations of this study are that it reported on only 2 cases and that a homemade system that is not commercially available was used.

The next study was published more than a decade later. ${ }^{13}$ In this study, the motor cortex of 10 patients with rolandic tumors was evaluated with a homemade nTMS system in which an electromagnetic navigation system was integrated with TMS for the purpose of positioning the TMS coil. The mean distance between hotspots of the 2 modalities was 3.4 (SD 3.0) $\mathrm{mm}$ (range $0-7 \mathrm{~mm}$ ). The limitation of this study was that the pre- and intraoperative mappings were performed in the same predefined 5-mm raster, so the resulting comparative data were not fully quantitative but were semiquantitative. This system is not commercially available either, so the applicability of the findings is limited.

The next study ${ }^{6}$ evaluated hotspots determined by nTMS and DES in 2 patients with brain tumors. In 1 patient the distance between hotspots was estimated at less than $5 \mathrm{~mm}$, but in the other case the comparison referred to the distance between hotspots by preoperative nTMS and DES that was conducted after tumor removal. So the latter data are unreliable because brain shift is likely to have occurred. This study also suffers from the very small sample size.

These first 3 studies all used self-made systems, were semiquantitative, and/or had a sample size that was too small from which to draw general conclusions about the spatial accuracy of nTMS. Since then 8 more studies have been published (up to October 2012-2 of these studies 3 ,19 were published online first in 2012 and then appeared in print in 2013). These studies contain larger clinical samples, and all but one ${ }^{3}$ were quantitatively evaluated. The challenge of visualizing the stimulation point at the "correct" (that is, the most likely) anatomical area where the stimulation would be effective, has been addressed differently by different systems. The first-generation nTMS systems projected the midpoint of the stimulation coil 
Use of nTMS in rolandic brain tumor surgery

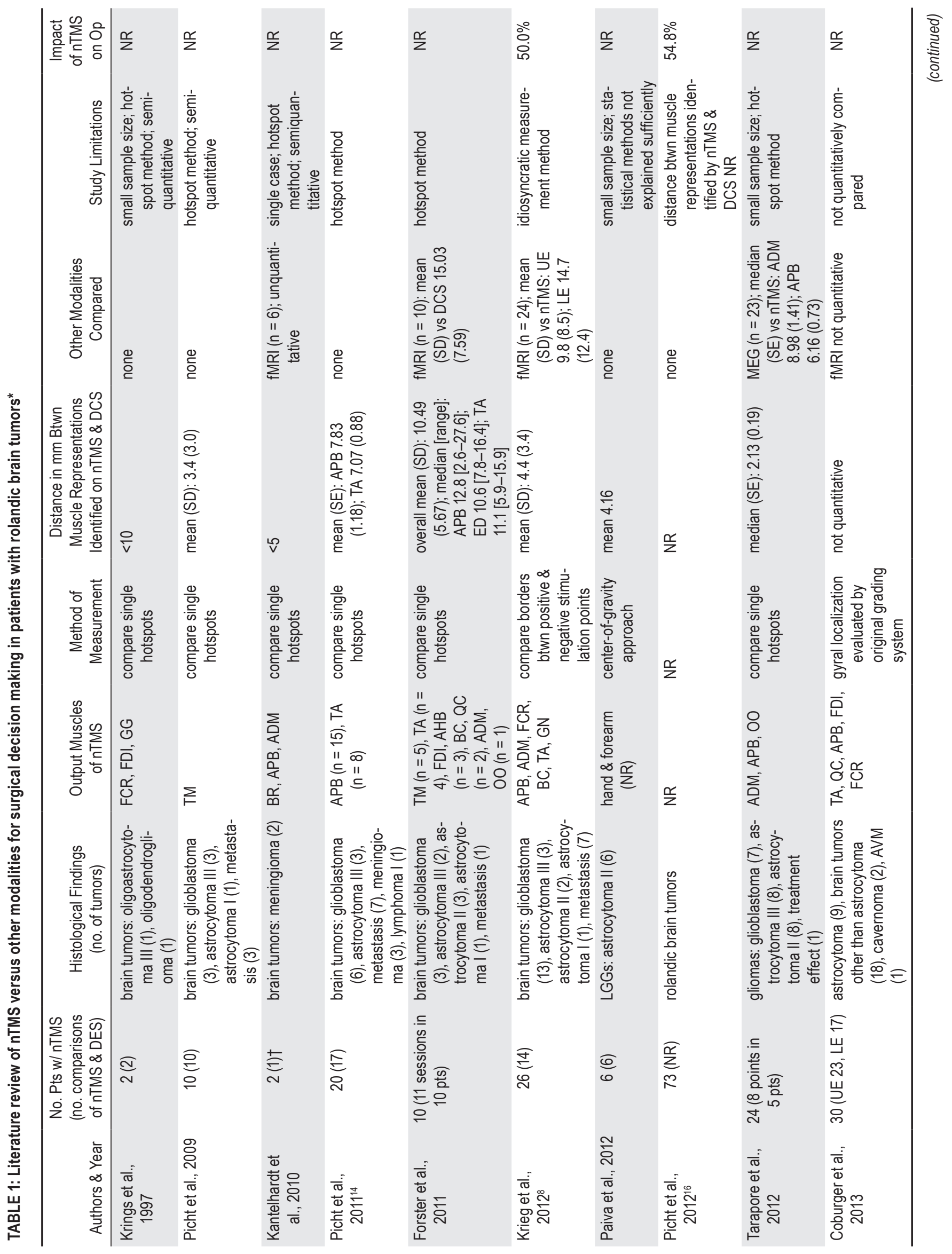




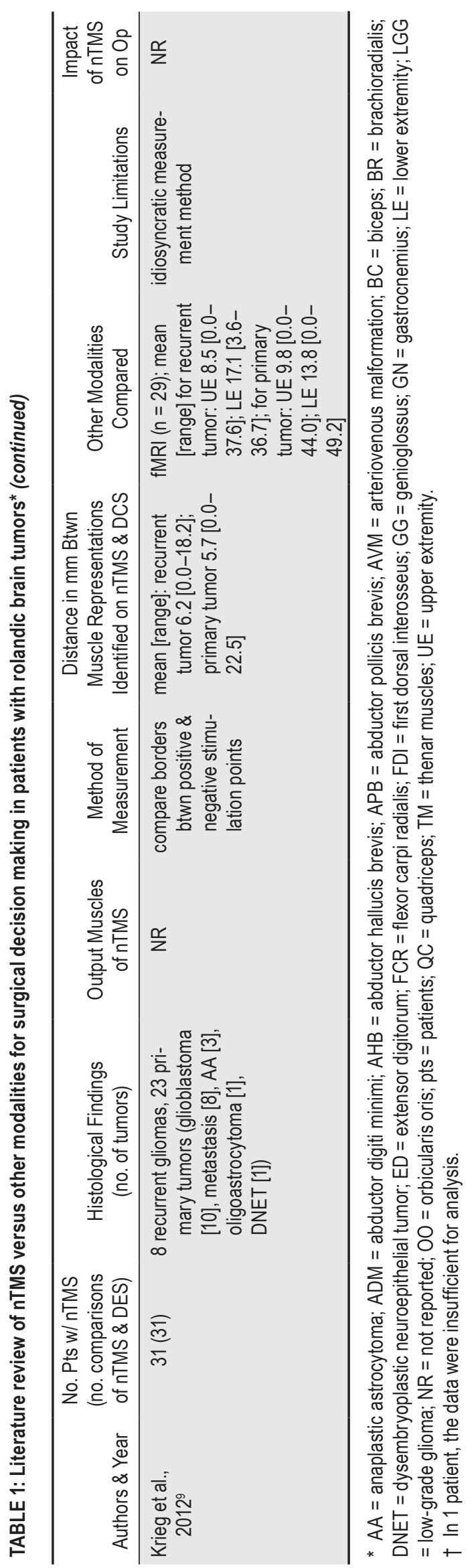

orthogonally onto the underlying cortex..$^{6,10,11,13}$ This approach ignored the significant effect that the individual cortical anatomy, the tilting angle, and the rotation of the coil have on the resulting effective "e-field" distribution. The more recent studies were conducted with a system that projects the calculated e-field induced by the magnetic pulse onto a 3D model of the brain, which is locally fitted with up to 40,000 spheres for e-field modeling. This approach assures a high accuracy in matching the virtual stimulation point to the "real" anatomy derived from the anatomical MRI scan. $3,4,8,9,14,21$

The first of the 8 studies $^{14}$ performed using a secondgeneration TMS system was in 20 patients with rolandic tumors, although only 17 had surgery and thus DES. In this study, DES locations were chosen independently of nTMS, and the distance between nTMS and DES hotspots was quantitatively determined. The mean (SE) distance between the nTMS and DES hotspots was $7.83(1.18) \mathrm{mm}$ for the APB muscle $(\mathrm{n}=15)$ and $7.07(0.88) \mathrm{mm}$ for the TA muscle $(\mathrm{n}=8)$. Importantly, the mean (SE) distance decreased to $4.70(1.09) \mathrm{mm}$ for APB $(\mathrm{n}=8)$ and 5.61 $(0.47) \mathrm{mm}$ for TA $(\mathrm{n}=5)$ after exclusion of the patients in whom possibly insufficient ( $<15$ stimulations) DES mapping was performed for these muscles. This study also reported comparisons on 3 other muscles in subsets of the sample.

In the same year, Forster et al. ${ }^{4}$ reported their experience with nTMS in 10 patients with rolandic tumors when compared with DES and fMRI. This study has been of particular interest because it first provided a simultaneous comparison of TMS, fMRI, and DES, thus enabling neurosurgeons to compare their options for preoperative mapping. The mean (SD) distance between the hotspots evaluated by nTMS and DES was 10.49 (5.67) mm (range $2.6-27.6 \mathrm{~mm}$ ). One problem with this calculation, though, was that the pairs of nTMS and DES hotspots compared were from 9 different muscles. Nonetheless, this result was smaller than the mean (SD) distance between the hotspots of fMRI and DES: 15.03 (7.59) mm (range 3.4$22 \mathrm{~mm}$ ). Therefore this study advocated that nTMS is better correlated to DES than fMRI. Yet, the use of different muscles may have accounted in part for the discrepancy between fMRI and nTMS findings: 5 hand/arm muscles, 3 leg muscles, and 1 facial muscle were recorded for TMS, whereas activation areas from the first dorsal interosseous muscle or toe movement were obtained for $\mathrm{fMRI}$. One other interesting finding from this study was that the median distance for the TA muscle relative to DES was larger for nTMS (11.1 $\mathrm{mm}$ [range 5.9-15.9 $\mathrm{mm}$ ]) than for fMRI (9.4 mm [range 5.7-19.1 mm]). These data suggested that nTMS may be less accurate for deeper-lying cortical regions, such as the cortical region corresponding to leg muscles.

Krieg et al. ${ }^{8}$ reported their experience in the use of nTMS presurgically for the resection of rolandic tumors. They performed preoperative nTMS in 14 patients with lesions located within or adjacent to the precentral gyrus and in 12 patients with lesions in the subcortical white matter motor tract. In the first patient group mentioned, they compared the borders between positive and negative stimulation points for nTMS and DES on axial slices by 
using recalibrated screenshots and BrainLAB iPlan Net Cranial 3.0.1. Although this method of comparing areas is theoretically more accurate than the hotspot method, it is very sensitive to the examination setup and is idiosyncratic. Using this method, the mean (SD) of the distance between borders for nTMS versus DES was 4.4 (3.4) mm (range 1.9-9.2 $\mathrm{mm}$ ). These investigators also evaluated the difference between borders delineating the primary motor cortex according to blood oxygen level-dependent data on fMRI studies and the mapping area identified by nTMS. The mean (SD) between nTMS and fMRI for this method was $9.8(8.5) \mathrm{mm}$ (range 5.3-39.7 $\mathrm{mm}$ ) for the upper extremity and 14.7 (12.4) $\mathrm{mm}$ (range 8.4-33.5 mm) for the lower extremity. They mentioned that their data demonstrate that nTMS correlates well with intraoperative DES, whereas nTMS and fMRI differed significantly from each other. No comparison between preoperative fMRI and DES was performed, so it remains difficult to say based on this study whether nTMS is more accurate than fMRI. This study first described the impact of preoperative brain mapping performed using nTMS on the surgery. They reported that preoperative nTMS showed a positive influence on the operative result in 5 of 14 cases, and even changed the operative strategy in 2 cases.

Another study focused on patients with relatively homogeneous brain tumors (that is, only patients with lowgrade glioma with a maximum diameter of $4 \mathrm{~cm}$ were included). Patients were assessed using a type of nTMS system that was only used in this study. ${ }^{11} \mathrm{~A}$ "center-ofgravity" approach was used to compare the difference between nTMS and DES. In theory, this method reflects better cortical representation of each muscle than the hotspot approach and should be encouraged in the future. The authors reported a mean distance between nTMS and DES of $4.16 \mathrm{~mm}$ (range 2.56-5.27 mm). The major limitations of this study were its small sample size $(n=6)$ and inadequate explanation of the statistical methods.

The next study, ${ }^{16}$ which included 73 patients with rolandic brain tumors, is of particular interest because it widely described the influence of nTMS on surgical planning. In this study the influence of nTMS was assessed prospectively by using a categorical ranking questionnaire. The authors found the influence of nTMS on the surgical planning to be as follows: it confirmed the expected anatomy in $21.9 \%$ of patients, added knowledge that was not used in $23.3 \%$, added awareness of high-risk areas in $27.4 \%$, modified the approach in $16.4 \%$, changed the planned extent of resection in $8.2 \%$, and changed the surgical indication in $2.7 \%$. Indeed, preoperative nTMS made an impact on $54.8 \%$ of the patients assessed, and in $27.3 \%$ of the patients nTMS even changed the surgical strategy (modified the surgical approach in $16.4 \%$, changed the planned extent of resection in $8.2 \%$, and changed the surgical indication in $2.7 \%$ ).

In the next study identified by our review, mapping was performed in 24 patients but then comparisons were made only in 5 , because positive motor sites were exposed only in 5 patients with the less invasive tailored craniotomy policy these investigators used. ${ }^{21}$ They calculated the difference between hotspots identified by nTMS and DES at 8 points in 5 patients as a median (SE) of $2.13(0.19)$ $\mathrm{mm}$. An interesting point in this study is that they reported that negative nTMS mapping correlates with negative DES mapping as well; in other words, DES mapping did not find any new motor sites where TMS had not. The study also included the result of a comparison between motor areas identified by nTMS and MEG. The median (SE) distance between the 2 hotspots of 46 sites in 23 patients was reported as $4.71(1.08) \mathrm{mm}$; unfortunately a comparison of MEG to DES was not reported.

Coburger et al. ${ }^{3}$ reported their experience comparing preoperative nTMS and fMRI with intraoperative DCS in 30 patients with brain tumors in or adjacent to the primary motor cortex. From the results of comparison of their own "accuracy score," they concluded that nTMS can be used more easily preoperatively compared with fMRI, and its cortical spatial resolution is more precise than fMRI. Their study included a large number of cases, and in contrast to all other studies they used a semiquantitative classification system with grades ranging from 1 to 4 , which described the spatial accuracy of nTMS in terms of identifying the M1 region. In the study, Grade 1 represents a distinct gyral localization, Grade 2 a projection over the adjacent sulcus, and Grade 3 a projection over the adjacent gyrus. Grade 4 characterizes an inability to localize the motor cortex. With this method, the accuracy was better in nTMS compared with fMRI both for upper and lower extremities. Their result suggested that nTMS represents a highly valuable supplement for preoperative brain mapping.

The latest report that was found by our review is a report by Krieg et al..$^{9}$ They reported the result of brain mapping by nTMS in 8 patients with recurrent gliomas and in 23 patients with initial operations for lesions in or adjacent to motor cortex for the purpose of prospectively evaluating nTMS accuracy in recurrent gliomas. Their study is of particular interest because they were the first to show that scarring, edema, and other effects of tumor surgery do not affect nTMS accuracy. In this study, contrary to the results of the study by Forster et al., ${ }^{4}$ the discrepancy between nTMS and DES for the lower extremity is smaller than that between fMRI and nTMS.

In summary, all studies reviewed here concluded that nTMS correlated well with the gold standard of DES. ${ }^{3,4,6,8-11,13,14,21}$ A total of 199 attempts in 161 patients to identify the motor cortex using nTMS were described. In only 1 patient, who had an infiltrating glioma within the somatosensory cortex, could TMS not identify any motor site. ${ }^{21}$ We have calculated the mean distance between motor cortex identified on nTMS and DES by using the mean distance described in 6 quantitatively evaluated studies. ${ }^{4,8,9,11,14,21}$ We have then weighted the mean from each study by the number of patients that mean was derived from. In 1 study, ${ }^{14}$ we used only the data for APB (n = 15) for simplicity. With this method, a weighted mean distance between nTMS and DES of $6.18 \mathrm{~mm}$ in 81 patients was calculated.

Two studies ${ }^{8,16}$ reported the impact of preoperative nTMS examination on the therapeutic decision making in a total of 87 patients. In $98.9 \%$ of cases reliable identification of functional tissue was documented after addition of nTMS. The surgical approach based on anatomical imag- 
ing alone was changed in $14(16.1 \%)$ of 87 patients after addition of the nTMS information, the planned extent of tumor resection was increased in $6(6.9 \%)$ of 87 patients, and the surgical indication was changed in $2(2.3 \%)$ of 87 patients.

\section{Discussion}

Preoperative functional brain imaging is now used widely in the context of rolandic brain tumor surgeries. The most widely adopted method is fMRI, but MEG, PET, and electroencephalography have also been used for preoperative mapping. ${ }^{12}$ These methods are all indirect. Furthermore, fMRI and PET rely on metabolic changes due to cortical activity after voluntary action, such as muscle contraction. ${ }^{15}$ Five studies addressed the spatial accuracy of fMRI in comparison with nTMS in identi-

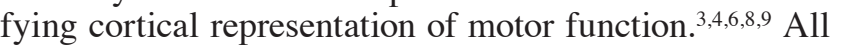
but one report stated a better correlation of nTMS than fMRI when compared with DES; only in the report by Forster et al. was the median distance for the TA muscle relative to DES larger for nTMS than for fMRI. This result raises the question whether nTMS may be less accurate for deeper-lying cortical regions, such as the cortical region corresponding to leg muscles. However, on the contrary, Coburger et al. ${ }^{3}$ reported (using their own accuracy scores) that nTMS showed statistically significantly higher spatial accuracy of the motor representation of the lower extremity than fMRI, and Krieg et al. ${ }^{8}$ also reported better spatial accuracy of nTMS for lower extremity over fMRI. Three studies reported hotspot distances between TMS and DCS separately for upper and lower extremities. ${ }^{4,11,14}$ In 24 comparisons the mean distance for upper extremity was $7.73 \mathrm{~mm}$ (range 0.42-27.6 $\mathrm{mm}$ ) and in 17 comparisons for lower extremity it was $9.47 \mathrm{~mm}$ (range 3.2-16.4 mm). Whether nTMS can identify the motor representation of the lower extremity with sufficient accuracy is currently still controversial and has to be addressed by future studies.

The spatial accuracy of nTMS for the mapping of hand and lower-arm muscles can be considered acceptable for surgical planning, as shown in the available reports..$^{3,4,6,8-11,13,14,21}$ It enables the surgeon to identify spatially accurate cortical representations of individual muscles vis-à-vis the gold standard of DES. Nevertheless, it has to be emphasized that nTMS is not a substitute for DES. Navigated TMS is performed outside the operating theater before and/or after operation, whereas DES is performed in the operating theater. This fact implies the following limitations and unique capabilities of each method: DES is an invasive method and its extent in area and time is limited by the surgical requirements. On the other hand, DES is the only modality that allows for direct identification of subcortical pathways and for monitoring of brain function at the time of surgery. The nTMS method provides information complementary to DES derived from its unique features. The overarching strength of nTMS is that it is so far the only mapping modality other than DES that is capable of stimulating brain and recording the output.

Navigated TMS can be performed repetitively preop- eratively and postoperatively, and according to the literature it has the following unique capabilities that supplement the information provided by intraoperative DES. 1) The nTMS method provides an objective assessment of the possibility of recovery of motor function. For example, in patients who have become plegic, nTMS can show if motor function is still possible..$^{15}$ 2) The nTMS method provides preoperative clarification of detailed cortical motor representation, which can result in smaller craniotomies that can prevent injury to eloquent tissue; for example, surgeons can apply keyhole approaches without exposing motor cortex. According to the results of 2 prospective studies, the surgical approach was changed in $14(16.1 \%)$ of 87 patients after nTMS mapping data were reviewed..$^{8,16,17}$ 3) The nTMS assessment can also be performed repeatedly across time, and this can enable visualization of plastic changes, which may influence the timing of the next surgical interventions; for example, eloquent tissue infiltrated by brain tumor that prevented total resection can become resectable due to shifting of the functional area over time..$^{20} 4$ ) The nTMS assessment enables an objective preoperative estimation of the extent of safe cortical tumor resection. According to the results of 2 prospective studies, preoperative brain mapping performed using nTMS led to a change of planned extent of tumor resection in $6.9 \%$ of the cases, and the surgical indication was changed in $2(2.3 \%)$ of 87 patients. $\left.{ }^{8,17} 5\right)$ The nTMS method can be used to define accurate "seedpoints" for diffusion tensor imaging, to visualize the pathways of the pyramidal fiber tracts. This approach can improve the accuracy of diffusion tensor imaging. ${ }^{5,7} 6$ ) The nTMS method is applicable also in small children; ${ }^{2}$ on the other hand, the modalities that require a patient's voluntary movement such as fMRI sometimes cannot be performed in small children or uncooperative or disabled patients. 7) Use of nTMS enables the surgeon to assess the functional status of the motor system in patients with brain tumor. For example, a high interhemispheric ratio of resting motor threshold or a low interhemispheric ratio of MEP amplitude was reported to suggest imminent deterioration of the patient's motor status. ${ }^{17}$ Altogether, these 7 unique capabilities of nTMS enable neurosurgeons to improve rolandic tumor resection through advanced planning and better postoperative evaluation of the surgery.

\section{Conclusions}

Navigated transcranial magnetic stimulation is the only mapping modality so far that is capable of stimulating the brain and recording the output in a noninvasive and painless way. Its accuracy for delineation of the motor cortex is comparable to that of DES. However, DES cannot be replaced by a noninvasive method due to its unique capability to stimulate subcortical structures accurately and to monitor function during surgery. The nTMS method is clinically beneficial not only by providing the same cortical spatial information as DES outside the operating theater, but in addition due to its capacity to provide information on the functional status of the motor system, to provide prognostic information with respect to functional outcome, to be applicable in plegic patients and young 
children, and to identify plastic changes of functional topography. Currently the application of nTMS for analysis of language-related areas is under intense investigation, with promising results. ${ }^{19}$

\section{Disclosure}

None of the authors has any personal financial or institutional interest in any of the drugs, materials, or devices described in this article. This work was supported by a grant from the Berliner Krebsgesellschaft.

Author contributions to the study and manuscript preparation include the following. Conception and design: all authors. Acquisition of data: Picht, Takahashi. Analysis and interpretation of data: Picht, Takahashi. Drafting the article: Picht, Takahashi. Critically revising the article: all authors. Reviewed submitted version of manuscript: all authors. Approved the final version of the manuscript on behalf of all authors: Picht. Statistical analysis: Takahashi. Study supervision: Vajkoczy.

\section{References}

1. Barker AT, Jalinous R, Freeston IL: Non-invasive magnetic stimulation of human motor cortex. Lancet 1:1106-1107, 1985

2. Coburger J, Karhu J, Bittl M, Hopf NJ: First preoperative functional mapping via navigated transcranial magnetic stimulation in a 3-year-old boy. Case report. J Neurosurg Pediatr 9:660-664, 2012

3. Coburger J, Musahl C, Henkes H, Horvath-Rizea D, Bittl M, Weissbach $\mathrm{C}$, et al: Comparison of navigated transcranial magnetic stimulation and functional magnetic resonance imaging for preoperative mapping in rolandic tumor surgery. Neurosurg Rev 36:65-75, 2013

4. Forster MT, Hattingen E, Senft C, Gasser T, Seifert V, Szelényi A: Navigated transcranial magnetic stimulation and functional magnetic resonance imaging: advanced adjuncts in preoperative planning for central region tumors. Neurosurgery 68:1317-1325, 2011

5. Frey D, Strack V, Wiener E, Jussen D, Vajkoczy P, Picht T: A new approach for corticospinal tract reconstruction based on navigated transcranial stimulation and standardized fractional anisotropy values. Neuroimage 62:1600-1609, 2012

6. Kantelhardt SR, Fadini T, Finke M, Kallenberg K, Siemerkus J, Bockermann V, et al: Robot-assisted image-guided transcranial magnetic stimulation for somatotopic mapping of the motor cortex: a clinical pilot study. Acta Neurochir (Wien) 152:333-343, 2010

7. Krieg SM, Buchmann NH, Gempt J, Shiban E, Meyer B, Ringel F: Diffusion tensor imaging fiber tracking using navigated brain stimulation-a feasibility study. Acta Neurochir (Wien) 154:555-563, 2012

8. Krieg SM, Shiban E, Buchmann N, Gempt J, Foerschler A, Meyer B, et al: Utility of presurgical navigated transcranial magnetic brain stimulation for the resection of tumors in eloquent motor areas. Clinical article. J Neurosurg 116:9941001,2012

9. Krieg SM, Shiban E, Buchmann N, Meyer B, Ringel F: Presurgical navigated transcranial magnetic brain stimulation for re- current gliomas in motor eloquent areas. Clin Neurophysiol [epub ahead of print], 2012

10. Krings T, Buchbinder BR, Butler WE, Chiappa KH, Jiang HJ, Rosen BR, et al: Stereotactic transcranial magnetic stimulation: correlation with direct electrical cortical stimulation. Neurosurgery 41:1319-1326, 1997

11. Paiva WS, Fonoff ET, Marcolin MA, Cabrera HN, Teixeira MJ: Cortical mapping with navigated transcranial magnetic stimulation in low-grade glioma surgery. Neuropsychiatr Dis Treat 8:197-201, 2012

12. Picht T, Atalay A: Preoperative motor mapping, in Hayat MA (ed): Tumors of the Central Nervous System. Dordrecht, Netherlands: Springer, 2012, Vol 4, pp 289-300

13. Picht T, Mularski S, Kuehn B, Vajkoczy P, Kombos T, Suess $\mathrm{O}$ : Navigated transcranial magnetic stimulation for preoperative functional diagnostics in brain tumor surgery. Neurosurgery 65 (6 Suppl):93-98, 2009

14. Picht T, Schmidt S, Brandt S, Frey D, Hannula H, Neuvonen $\mathrm{T}$, et al: Preoperative functional mapping for rolandic brain tumor surgery: comparison of navigated transcranial magnetic stimulation to direct cortical stimulation. Neurosurgery 69:581-588, 2011

15. Picht T, Schmidt S, Woitzik J, Suess O: Navigated brain stimulation for preoperative cortical mapping in paretic patients: case report of a hemiplegic patient. Neurosurgery 68: E1475E1480, 2011

16. Picht T, Schulz J, Hanna M, Schmidt S, Suess O, Vajkoczy P: Assessment of the influence of navigated transcranial magnetic stimulation on surgical planning for tumors in or near the motor cortex. Neurosurgery 70:1248-1257, 2012

17. Picht T, Strack V, Schulz J, Zdunczyk A, Frey D, Schmidt S, et al: Assessing the functional status of the motor system in brain tumor patients using transcranial magnetic stimulation. Acta Neurochir (Wien) 154:2075-2081, 2012

18. Sanai N, Berger MS: Intraoperative stimulation techniques for functional pathway preservation and glioma resection. Neurosurg Focus 28(3):E1, 2010

19. Sollmann N, Picht T, Makela JP, Meyer B, Ringel F, Krieg SM: Navigated transcranial magnetic stimulation for preoperative language mapping in a patient with a left frontoopercular glioblastoma. Case report. J Neurosurg 118:175-179, 2013

20. Takahashi S, Jussen D, Vajkoczy P, Picht T: Plastic relocation of motor cortex in a patient with LGG (low grade glioma) confirmed by NBS (navigated brain stimulation). Acta Neurochir (Wien) 154:2003-2008, 2012

21. Tarapore PE, Tate MC, Findlay AM, Honma SM, Mizuiri D, Berger MS, et al: Preoperative multimodal motor mapping: a comparison of magnetoencephalography imaging, navigated transcranial magnetic stimulation, and direct cortical stimulation. Clinical article. J Neurosurg 117:354-362, 2012

Manuscript submitted January 3, 2013.

Accepted January 28, 2013.

Please include this information when citing this paper: DOI: 10.3171/2013.1.FOCUS133.

Address correspondence to: Thomas Picht, M.D., Department of Neurosurgery, Charité-Universitätsmedizin, Augustenburger Platz 1, Berlin 13353, Germany. email: thomas.picht@charite.de. 